\title{
Gestão do trabalho: desenho organizacional, processo estratégico e tipos de trabalho ${ }^{1}$
}

\author{
Rosalia Aldraci Barbosa LaVARDa \\ Universidade Federal de Santa Catarina / Departamento de CiênCIas da Administração, Centro Sócio Econômico, \\ FLORIANÓPOLIS - SC, BRASIL \\ Carlos Eduardo facin lavarda \\ Universidade Federal de SANTA Catarina / Departamento de CIÊnCIAS ContábeIS, CEnTro Sócio Econômico, \\ FLORIANÓPOLIS - SC, BRASIL
}

\begin{abstract}
Resumo
O objetivo deste estudo é verificar, dentro da gestão do trabalho, como os componentes do desenho e processo estratégico organizacionais se relacionam com os tipos de trabalho desempenhados pela organização. Foi realizada uma pesquisa qualitativa com estudo de caso. As técnicas de coleta de dados foram entrevistas semiestruturadas, análise de documentos e observação direta. A análise de dados baseou-se em pattern-matching e narrativa. Os resultados apontam que, mesmo que as características de cada tipo de trabalho estejam presentes nos casos analisados, há indícios de que novas variáveis precisam ser pesquisadas para explicar a variação dos níveis de racionalidade e a participação no processo estratégico para configurar as diferentes formas de gestão do trabalho.
\end{abstract}

Palavras-chave: Desenho organizacional. Processo estratégico. Gestão do trabalho. Metodologia qualitativa.

\section{Work management: organizational design, strategic process, and work types}

\section{Abstract}

This study aims to verify, within work management, how the components of organizational design and strategic process relate to the work types performed by the organization. We conducted a qualitative research with case study. The data collection techniques were semi-structured interviews, analysis of documents, and direct observation. Data analysis was based on pattern-matching and narrative. The results point out that, although the characteristics of each work type are observed in the cases under analysis, there is evidence that new variables require investigation to explain variation in the rationality levels and participation in the strategic process to configure the various ways of work management.

Keywords: Organizational design. Strategic process. Work management. Qualitative methodology.

\section{Gestión del Trabajo: diseño organizativo, proceso estratégico y tipos de trabajo}

\section{Resumen}

El objetivo del estudio es determinar si el diseño y el proceso estratégico de la organización se relacionan con el tipo de trabajo desarrollado por ésta. Se realizó una investigación cualitativa a través de estudios de casos. Las técnicas empleadas para la recolección de los datos fueron las entrevistas semi-estructuradas, el análisis de documentos y la observación directa. El análisis de los datos se basó en el pattern-matching y narrativa. Los resultados indican que aunque las características de cada tipo de trabajo estén presentes en los casos analizados, es necesario profundizar en el estudio de nuevas variables para explicar la variación en los niveles de la racionalidad y la participación en el proceso estratégico para configurar las diferentes formas de gestión del trabajo.

Palabras clave: Diseño organizativo. Proceso estratégico. Gestión del trabajo. Metodología cualitativa.

${ }^{1}$ Apoio do Conselho Nacional de Desenvolvimento Científico e Tecnológico- CNPq 


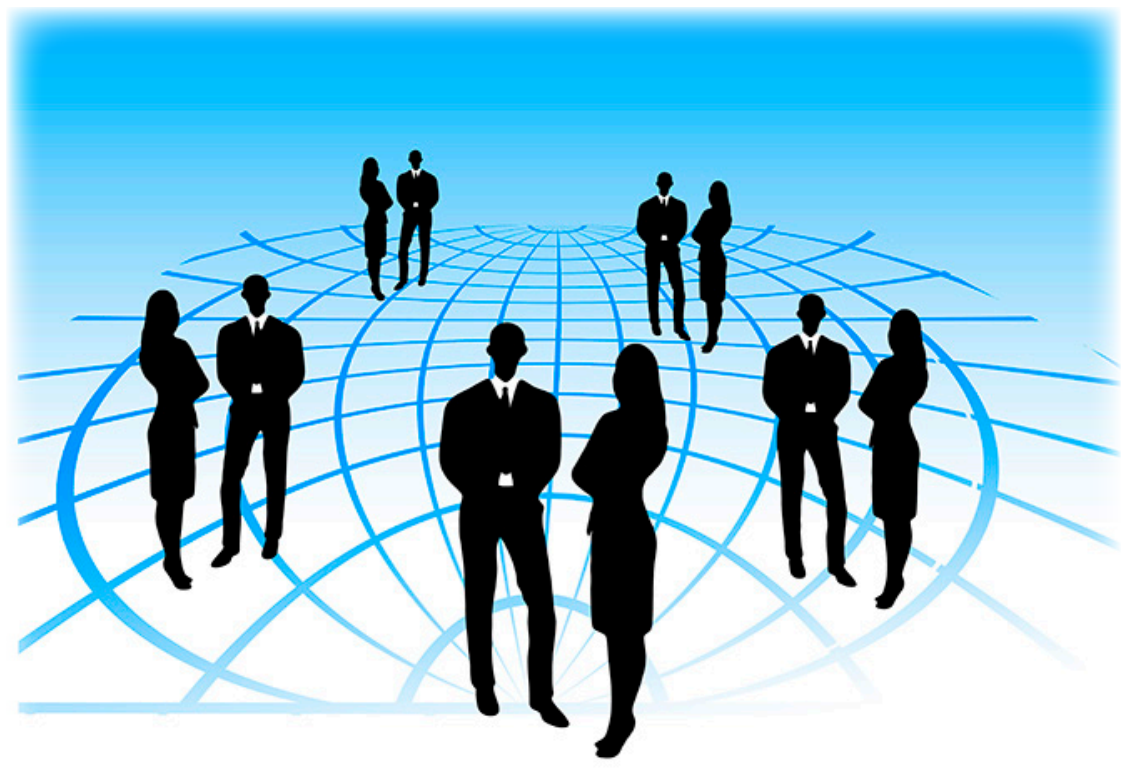

\section{INTRODUÇÃO}

A gestão do trabalho realizado nas organizações parece estar relacionada à forma com que a mesma se ajusta às condições do ambiente e a suas escolhas estratégicas, de acordo com os estudos de Child (1997). Isso sugere que a estratégia é uma ferramenta voluntária e deliberada nas mãos da direção, que conduz a escolha e a definição dos fatores organizacionais, determinando como a organização vai atuar e do que necessita para tal, escolhendo distintos caminhos para alcançar os objetivos previamente definidos (LAVARDA, CANET e PERIS, 2011).

Seguindo esse entendimento, o objetivo desta pesquisa é verificar, dentro da gestão do trabalho, como os componentes do desenho e do processo estratégico organizacionais se relacionam com os tipos de trabalho desempenhados pela organização.

Entende-se por componentes do desenho organizacional a centralização, a formalização, as políticas de gestão de pessoas e o nível de conhecimento para realização do trabalho (MORENO, PERIS e GONZÁLEZ, 2001). Também se entende por componentes do processo estratégico, o nível de racionalidade, visão, participação e flexibilidade, segundo Fredrickson e Mitchell (1984), Mintzberg e Waters (1985), Hamel e Välikangas (2003), Andersen (2004), Elbana (2006) e Lavarda, Canet e Peris (2010a; 2010b; 2011).

Os tipos de trabalho desempenhados pela organização são classificados, segundo Perrow (1970), como: trabalho rotineiro e repetitivo; trabalho não rotineiro de qualificação média/baixa; trabalho profissional e trabalho qualificado, inovador ou criativo.

Assim, considerando a relevância de estudar a relação entre tipos de trabalho (de rotineiro a inovador/criativo), a partir do enfoque do desenho organizacional e do processo estratégico, surge a seguinte questão de pesquisa para se investigar: como os componentes do desenho e do processo estratégico organizacionais se relacionam com os tipos de trabalho desempenhados pela organização?

Para responder a essa questão, realizamos uma pesquisa qualitativa por meio de estudo de casos em três organizações de setores específicos (indústria e serviços). As técnicas de coleta de dados foram entrevistas semiestruturadas, análise de documentos e observação direta. A análise dos dados baseou-se na análise de padrão do comportamento (pattern-matching) de

\footnotetext{
* Fonte da imagem: Disponível em: <https://pixabay.com/pt/rede-pessoa-par-mulher-homem-889354/ >. Acesso em 17 mar. 2016
} 
Trochim (1989) e Pérez-Aguiar (1999) e análise narrativa seguindo a Godoi, Bandeira-de-Mello e Silva (2006). Assim, passamos a revisar esses dois campos teóricos: o que explica os tipos de trabalho e o que trata sobre o processo de formação da estratégia.

\section{CARACTERIZAÇÃO DOS TIPOS DE TRABALHO}

Revisando a classificação original dos tipos de trabalho de Perrow (1967) e apresentada por Cury (2000, p. 74) e Daft (2002, p. 199-201), encontramos a classificação de quatro tipos de trabalho, definidos como: a) artesanato - não analisável ou de difícil medição e com poucas situações excepcionais; b) não rotineiro - não analisável e com muitas situações excepcionais; c) rotineiro - produção de item padronizado, que apresenta pouca variedade de problemas, é analisável, ou seja, está formalizado, é possível medir (ALCHIAN e DEMSETZ, 1972) e controlar e apresenta poucas situações excepcionais; d) Engenharia apresenta alto grau de variabilidade, muitas situações excepcionais e é inovador.

Dado que o trabalho realizado apresenta diferentes características, como consequência dos produtos e serviços que a empresa desenvolve e dado que os diferentes tipos de trabalho condicionam a forma de administrar a organização, apresentamos uma versão modificada do modelo de tipos de trabalho de Perrow $(1967 ; 1970)$, que relaciona os mesmos com categorias de análise organizacionais que são: centralização (C), formalização (F), políticas de gestão de pessoas (GP) e nível de conhecimento ou especialização (K).

Considerando as categorias de análise C, F, GP e K, destacamos que a centralização (C) pode ser estrutural, entre os diferentes níveis hierárquicos; ou relacionada com a supervisão do trabalho, pois "o nível de centralização ou descentralização de uma organização depende do nível em que se delega capacidade para tomar decisões nos níveis hierárquicos inferiores e nas diferentes áreas ou unidades da organização" (MORENO, PERIS e GONZÁLEZ, 2001, p. 217).

A formalização (F) refere-se ao planejamento dos sistemas organizacionais ou ao modo pelo qual se regula o conteúdo do trabalho; abrangendo as regras não escritas que orientam e canalizam de forma suficientemente clara o comportamento, os procedimentos ou processos padronizados, não documentados, e as regras e procedimentos escritos aos quais devem ajustar-se as atividades da empresa.

As políticas de gestão de pessoas (GP) podem ser funcionais, relacionadas ao recrutamento, seleção e capacitação; ou complementares, que correspondem às formas de medição, avaliação e controle de desempenho, assim como, os sistemas de recompensa e incentivos. Destaca-se que o pagamento de incentivos deve estar presente quando a dificuldade e os custos de fiscalização são elevados para verificar o desempenho do trabalhador (MORENO, PERIS e GONZÁLEZ, 2001).

A caracterização dos tipos de trabalho, sua análise e controle está relacionada com o nível de conhecimento ou especialização (K) que o trabalhador incorpora: conhecimento específico ou conhecimento detalhado (difícil de transmitir); conhecimento tácito, também classificado como especialização do trabalho (NONAKA e TAKEUCHI, 1997).

Nesse sentido, estabelecemos, para o desenvolvimento deste estudo, como propõem Moreno, Peris e González (2001) e Lavarda, Canet e Peris (2011), quatro tipos de trabalho: T1 - trabalho rotineiro e repetitivo; T2 - trabalho não rotineiro de especialização média/baixa; T3 - trabalho profissional e T4 - trabalho qualificado, inovador ou criativo, conforme apresentado na Figura 1.

O trabalho rotineiro ou repetitivo (T1) é um tipo de trabalho não submetido a exceções ou mudanças e é de fácil análise/controle, não incorpora conhecimento específico relevante e requer baixa especialização (-K). Como consequência, a ausência de mudanças permite um bom ajuste com uma formalização alta, mediante normas, procedimentos padronizados e regras $(+F)$, e uma centralização também alta $(+C)$ como consequência da fácil análise/controle, por exemplo, o trabalho de linha de produção.

O trabalho não rotineiro de qualificação média/baixa (T2) caracteriza-se por sua média ou baixa qualificação e suas mudanças frequentes. Não incorpora conhecimento específico relevante (-K), o que permite sua centralização ou supervisão a níveis médios ou altos $(+C)$ e está pouco formalizado $(-F)$ para que possa adaptar-se. As políticas funcionais de gestão de pessoas são mais importantes que as políticas complementares (-GP), por exemplo, o trabalho de atendimento a clientes. 
O trabalho profissional (T3) não está submetido a mudanças frequentes, é de difícil análise/controle e possui um grau de conhecimento relevante ao incorporar preparação profissional com a experiência (+K). Consequentemente, a centralização é baixa $(-\mathrm{C})$ e o trabalho está formalizado $(+\mathrm{F})$, como consequência dos protocolos e procedimentos que caracterizam o trabaIho profissional. Nesse tipo de trabalho, é conveniente fomentar o compromisso do trabalhador mediante políticas complementares de gestão de pessoas que melhorem os incentivos (+GP), por exemplo, o trabalho do médico, engenheiro, professor.

O trabalho qualificado e inovador ou criativo (T4) é um trabalho submetido a mudanças e de difícil análise/controle. Possui alto grau de conhecimento geral e de conhecimento específico, é altamente especializado (+K), representando formas de trabalho com muitos conhecimentos tácitos (artesãos muito qualificados, cientistas), profissionais ou diretores cujo trabaIho tem uma dimensão relevante de criatividade ou inovação. Esse tipo de trabalho não está centralizado (-C), visto que é de difícil análise, e está pouco formalizado (-F), já que deve existir um bom ajuste com as mudanças inerentes à criatividade e inovação. As políticas complementares de gestão de pessoas são extremamente importantes (+GP), pois, quando o trabalho é complexo e de difícil medição e controle, requer um reforço nas políticas de incentivo.

Figura 1

\section{Componentes do desenho organizacional}

\begin{tabular}{|c|c|c|c|}
\hline \multicolumn{4}{|c|}{ DESENHO ORGANIZACIONAL } \\
\hline T2 & $+\mathrm{C}$ & T1 & \\
\hline $\begin{array}{l}\text { Não Rotineiro } \\
\text { Baixa Formalizacão (-F) }\end{array}$ & & $\begin{array}{l}\text { Rotineiro } \\
\text { Alta Formalizacão }(+F)\end{array}$ & \\
\hline Alta Centralização (+C) & & Alta Centralização (+C) & \\
\hline Baixa Especialização (-K) & & Baixa Especialização (-K) & \\
\hline Baixa Gestão de Pessoas (-GP) & & Baixa Gestão de Pessoas (-GP) & \\
\hline \multicolumn{4}{|l|}{$-F$} \\
\hline T4 & & T3 & \\
\hline Criativo e Inovador & & Profissional & \\
\hline Baixa Formalização (-F) & & Alta Formalização (+F) & \\
\hline Baixa Centralização (-C) & & Baixa Centralização (-C) & \\
\hline Alta Especialização (+K) & & Alta Especialização (+K) & \\
\hline Alta Gestão de Pessoas (+GP) & & Alta Gestão de Pessoas (+GP) & \\
\hline & $-C$ & & \\
\hline
\end{tabular}

Fonte: Adaptado de Lavarda, Canet e Peris (2010b, p. 119).

Considerando os diferentes tipos de trabalho, verificamos que os mesmos estarão diretamente relacionados com a escolha estratégica (CHILD, 1997) e também com os objetivos organizacionais estabelecidos a partir da referida escolha. Assim, passamos a revisar como ocorre o processo de formação da estratégia organizacional, considerando como variáveis que compõem esse processo: racionalidade $(\mathrm{R})$, visão $(\mathrm{V})$, participação $(\mathrm{P})$ e flexibilidade $(\mathrm{Fx})$, caracterizando um processo mais deliberado ou mais emergente de formação da estratégia (MINTZBERG e WATERS, 1985).

\section{O PROCESSO ESTRATÉGICO}

Estratégia deliberada ocorre quando as estratégias pretendidas e planejadas são realizadas, já estratégia emergente é aquela na qual o padrão realizador não era expressamente pretendido, é uma estratégia que surge de um padrão que não estava 
previsto, estabelecido (MINTZBERG e WATERS, 1985). A principal diferença entre estratégia deliberada e emergente está em se considerar o foco, a direção e o controle. Na estratégia deliberada, a ênfase está na direção central e nas hierarquias, focando o controle. Na estratégia emergente, a ênfase está na ação coletiva e comportamentos convergentes, focando o aprendizado, ou seja, é a estratégia na prática (JARZABKOWSKI, 2003; WHITTINGTON, 2006; JARZABKOWSKI, BALOGUN e SEIDL, 2007; 2010; FENTON e LANGLEY, 2011; BALOGUN, JACOBS, JARZABKOWSKI et al., 2013). O processo estratégico tende a seguir um padrão integrador, com um equilíbrio entre racionalidade e as categorias de análise mais emergentes, como participação e flexibilidade (LAVARDA, CANET e PERIS, 2010b).

Nesse sentido, o conceito que destacamos como fundamental para este estudo é o de processo de formação da estratégia ou processo estratégico, concretamente, considerando o "como" esta deve ser desenvolvida. Tal processo tem sido investigado de diferentes perspectivas (MINTZBERG, 1973; FARJOUN, 2002; ANDERSEN, 2004; BULGACOV, SOUZA, PROHMANN et al., 2007).

Neste estudo, a relevância está na análise da perspectiva integradora do processo de formação da estratégia, ou seja, a soma da estratégia planejada ou racional com a estratégia mais emergente ou intuitiva, tal como refletem as contribuições de Mintzberg e Waters (1985), Hart (1992), Hamel e Prahalad (1994), Johnson, Melin e Whittington (2003), Andersen (2004), Elbanna (2006) e Lavarda, Canet e Peris (2011).

A relação entre o tipo de trabalho e a estrutura que a organização deve ter (desenho organizacional), é uma consequência do ajuste necessário entre as características do trabalho e a forma pela qual é organizado e dirigido. Entende-se que a explicação de por que se organiza de uma ou de outra maneira corresponde à visão da direção e a como essa se materializa num conjunto de produtos e serviços com os quais se responde ao ambiente, uma vez escolhido o mesmo, segundo a escolha estratégica de Child (1997).

Assim, para analisar se o processo estratégico é mais deliberado ou mais emergente, os componentes do processo estratégico envolvem o nível de racionalidade (R), visão (V), participação (P) e flexibilidade (Fx), segundo Fredrickson e Mitchell (1984), Mintzberg e Waters (1985), Hamel e Välikangas (2003), Andersen (2004), Elbana (2006) e Lavarda, Canet e Peris (2011).

A racionalidade implica identificação, avaliação e escolha de alternativas viáveis, no processo de tomada de decisões (FREDRICKSON e MITCHELL, 1984). Ela pode ser encontrada nos níveis de planejamento formal do plano estratégico e planos operacionais, definições e implementação de prazos, processo baseado em ferramentas e técnicas de análise (ELBANA, 2006).

A visão compartilhada pode ser compreendida como a capacidade do líder para transmitir a sua intuição de como desenvolver os processos, a importância de desenvolver uma inovação ou atingir um novo mercado; e em que medida o líder visionário é capaz de contagiar os membros de sua organização; a necessidade de implementar determinada ideia ou visão particular, integrando a racionalidade e a participação (MINTZBERG e WATERS, 1985).

A participação é o resultado do grau de envolvimento dos indivíduos e grupos de diferentes níveis hierárquicos, por meio de suas iniciativas e propostas na formulação da estratégia, segundo Golsorkhi, Rouleau, Seidl et al. (2010) e Lavarda, Canet e Peris (2011). O grau de autoridade distribuída, caracterizada pela delegação de tomada de decisões (baixo nível de centralização) e incentivos à participação (muitas vezes dependente das políticas complementares) também são aspectos importantes a considerar quando se observa a participação.

Já a flexibilidade, a partir do conceito de resiliência de Hamel e Välikangas (2003), é a capacidade dinâmica de reinventar, de mudar constantemente para se adaptar a novas situações. É importante para promover o ajuste interno necessário para responder às demandas ou mudanças do ambiente externo.

Assim, para a gestão do trabalho com características de T1 e T2 (rotineiros e pouco submetidos à mudança), entendemos que exigem alto nível de racionalidade (+R), baixa participação (-P), a visão praticamente não é conhecida pelos trabalhadores $(-\mathrm{V})$ e a flexibilidade no posto de trabalho é baixa $(-\mathrm{Fx})$.

Para administrar o trabalho com as características de T3 e T4 (profissional e inovador), ao contrário, exigem que o nível de racionalidade seja baixo $(-R)$, a visão seja compartilhada e comunicada $(+V)$, a participação no processo de formação da estratégia seja alta (+P) e a flexibilidade à mudança seja elevada (+Fx), conforme indica a Figura 2. 
Figura 2

\section{Componentes do processo de formação da estratégia}

\begin{tabular}{|c|}
\hline PROCESSO DE FORMAÇÃo ESTRATÉGICA \\
\hline T1 e T2 \\
Alta Racionalidade (+R) \\
Baixa Visão (-V) \\
Baixa Participação (-P) \\
Baixa Flexibilidade (-Fx) \\
T3 e T4 \\
Baixa Racionalidade (-R) \\
Alta Visão (+V) \\
Alta Participação (+P) \\
Alta Flexibilidade (+Fx)
\end{tabular}

Fonte: Elaborado pelos autores.

A partir do marco teórico revisado e buscando-se responder à questão de pesquisa, a proposição $(\mathrm{P})$ que orienta o estudo empírico é: a organização apresenta os componentes do desenho organizacional e da estratégia de acordo com os tipos de trabalho que desempenha, sendo que a simplicidade ou complexidade do trabalho determinam o adequado nível de ajuste. Mais especificamente:

$P_{a}$ : Para T1 rotineiro: altos níveis de formalização e de centralização; altas políticas funcionais e baixas políticas complementares de gestão de pessoas e baixa especialização - ajustam-se com alta racionalidade e baixa participação, visão e flexibilidade (estratégia deliberada);

$\mathbf{P}_{b}$ : Para T2 não rotineiro: médios níveis de formalização; alta centralização; altas políticas funcionais e baixas políticas complementares de gestão de pessoas e baixa ou média especialização - ajustam-se com alta racionalidade e baixa participação, visão e flexibilidade (estratégia deliberada);

$\mathbf{P}_{c}$ : Para T3 profissional: médios níveis de formalização; baixa centralização; altas políticas funcionais/complementares de gestão de pessoas e alta especialização - ajustam-se com baixa racionalidade e alta participação, visão e flexibilidade (estratégia emergente/integradora);

$\mathbf{P}_{\mathrm{d}}$ : Para T4 inovador: baixos níveis de formalização; baixa centralização; altas políticas funcionais/complementares de gestão de pessoas e alta especialização - ajustam-se com baixa racionalidade e alta participação, visão e flexibilidade (estratégia emergente/integradora); conforme Figura 3. 
Figura 3

Relação entre componentes da estratégia e do desenho organizacional segundo os tipos de trabalho

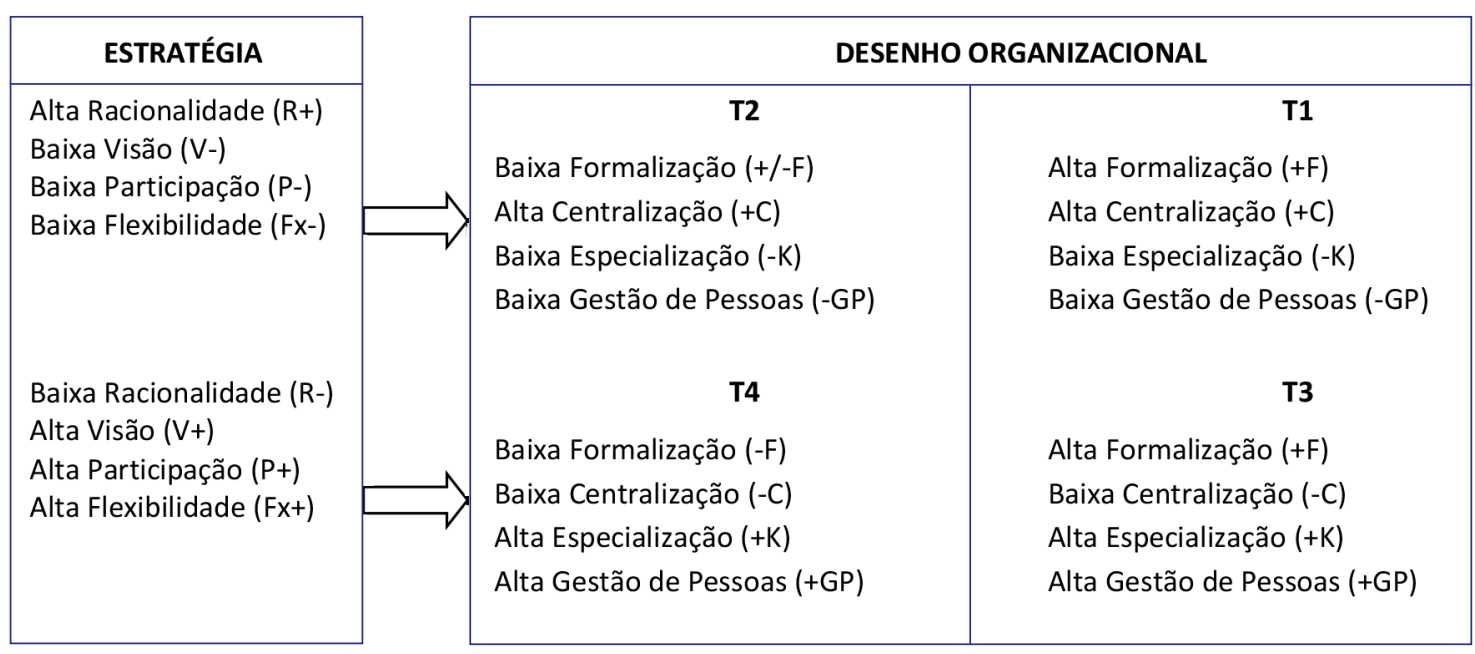

Fonte: Elaborado pelos autores.

\section{METODOLOGIA}

Para responder à questão de investigação, desenvolvemos uma pesquisa qualitativa (DENZIN e LINCOLN, 2006) usando a metodologia de estudo de casos múltiplos (EISENHARDT, 1989; YIN, 2005). Para alcançar os resultados pretendidos, foram adotadas como técnicas de coleta de dados: entrevistas semiestruturadas, análise de documentos (organograma, formulários, planejamento estratégico, página web) e observação direta. As três técnicas propiciaram a confirmação dos dados coletados, por meio da triangulação dos dados.

Os critérios para seleção das três organizações (EISENHARDT, 1989) basearam-se no porte, sendo especificados como de médio ou grande porte (a especificação do porte é dada pela necessidade de se encontrar um processo estratégico, uma área de $\mathrm{RH}$ e atividades de produção de bens ou serviços, devidamente formalizados como condição prévia para o desenvolvimento do estudo); além desse critério, buscamos organizações com características de exercer diferentes tipos de trabaIho, como organizações de setores específicos (saúde e educação) e que fossem localizadas no Vale do Itajaí - SC, seguindo o critério de acessibilidade.

Assim, chegamos ao Caso A, uma organização hospitalar, que é referência nacional em determinados procedimentos; Caso B, uma empresa têxtil que vem ganhando espaço cada vez maior no mercado nacional e internacional; Caso C, uma instituição federal de ensino e pesquisa, criada em 2008, com importante atuação em todo o Estado de Santa Catarina. Foram entrevistados ao todo quinze colaboradores, sendo um do nível operacional (somente para confirmação dos dados coletados nos níveis superiores e nas observações in loco), dois do tático e dois diretores do nível estratégico (alta direção) de cada organização. A duração das entrevistas foi de $5 \mathrm{~h}$ em cada organização, durante o período de março a junho de 2012. Todas as entrevistas foram gravadas, com o prévio consentimento da organização, para posterior análise dos dados coletados. Destacamos que o número de entrevistas está de acordo com a intensidade das respostas (CHARMAZ, 2009), sendo que a coleta de dados qualitativos não necessita de quantidade, mas sim de profundidade.

As organizações apresentaram seus organogramas, formulários, relatórios de produção e serviços e plano de desenvolvimento institucional, que propiciaram a análise da formalização. As visitas realizadas nas empresas propiciaram a observação do fluxo de trabalho e a observação de rotinas e procedimentos administrativos e de produção. 
Como unidade de análise, considerou-se a unidade principal ou matriz de cada uma das organizações. Como nível de análise, o nível micro-organizacional das organizações foi considerado para análise, formado por seus diferentes tipos de trabalho, o modo como são administrados e como é o processo de formação da estratégia.

Para analisar os resultados do estudo qualitativo, apresenta-se, no Quadro 1, a descrição das categorias de análise (CA), com seus fatores de medição e respectivos conceitos. Pretendemos, a partir dos fatores de medição, responder à proposição inicial deste trabalho, verificando se os dados ou fatos observados na prática se encaixam com as proposições formuladas teoricamente.

Assim, a análise dos dados foi baseada na técnica pattern matching (TROCHIM, 1989), utilizada na comparação de acontecimentos, comportamentos e situações encontradas na realidade estudada (por meio dos dados coletados nas entrevistas, análise de documentos e observação direta) com o marco teórico revisado e estruturado por meio das proposições teóricas (PÉREZ-AGUIAR, 1999). Conjuntamente, foi adotada a análise narrativa (GODOI, BANDEIRA-DE-MELO e SILVA, 2006), que possibilitou constatar, por meio do depoimento dos entrevistados, a confirmação ou evidência das categorias de análise (KERLINGER, 1979).

\section{Quadro 1}

\section{Relação entre categorias de análise, fatores de medição e seus conceitos}

\begin{tabular}{|c|c|c|}
\hline $\begin{array}{l}\text { CATEGORIAS } \\
\text { DE ANÁLISE } \\
\text { (CA) }\end{array}$ & $\begin{array}{l}\text { FATORES DE } \\
\text { MEDIÇÃO }\end{array}$ & CONCEITOS \\
\hline \multirow{4}{*}{$\begin{array}{l}\text { CA1 - Desenho } \\
\text { Organizacional }\end{array}$} & Centralização & $\begin{array}{l}\text { O quanto o trabalhador é autônomo no seu âmbito de trabalho e } \\
\text { corresponde também à centralização nos diferentes níveis } \\
\text { hierárquicos. }\end{array}$ \\
\hline & Formalização & $\begin{array}{l}\text { Programação e estruturação das atividades, regras escritas e não } \\
\text { escritas. Procedimentos referidos à gestão do trabalho. }\end{array}$ \\
\hline & $\begin{array}{l}\text { Políticas de } \\
\text { Gestão de Pessoas }\end{array}$ & $\begin{array}{l}\text { Seleção e capacitação de pessoal. E forma de medição, avaliação e } \\
\text { controle do desempenho (sistema de recompensas e incentivos). }\end{array}$ \\
\hline & Especialização & $\begin{array}{l}\text { É o nível de conhecimento exigido do trabalhador, para } \\
\text { desempenhar determina da tarefa/atividade. }\end{array}$ \\
\hline \multirow{4}{*}{$\begin{array}{l}\text { CA2 - Formação da } \\
\text { Estratégia }\end{array}$} & Racionalidade & Nível de formalidade e tomada de decisões. \\
\hline & Visão & Transmissão de valores. \\
\hline & Participação & Autoridade distribuída e participação nas decisões. \\
\hline & Flexibilidade & Capacidade de inovação e de adaptação à mudança. \\
\hline
\end{tabular}

Fonte: Elaborado pelos autores.

\section{ANÁLISE DOS RESULTADOS E DISCUSSÃO}

Inicialmente, apresentamos as evidências para cada categoria de análise referente a cada um dos tipos de trabalho de cada caso. Posteriormente, apresentamos a discussão da questão de pesquisa com as proposições teóricas (análise pattern-matching). Com o intuito de se apresentarem as categorias de análise com as evidências encontradas na organização, utilizaram-se, essencialmente, as entrevistas semiestruturadas (análise da narrativa). A análise dos documentos e observação direta é apresentada durante a análise das entrevistas, de forma complementar e confirmatória.

\section{CARACTERIZAÇÃo DOS CASOS E EVIDÊNCIAS DAS CATEGORIAS DE ANÁLISE}

A organização hospitalar analisada, Caso A, possui uma estrutura hierárquica formalizada, seu organograma está dividido em seis níveis de tomada de decisão, sendo o primeiro nível composto pelo conselho de administração (órgão deliberativo 
formado por conselheiros que não está operacionalmente na empresa); o segundo nível, pelo Diretor Superintendente; o terceiro nível, pelos demais diretores (médico, assistencial, negócios e administrativo financeiro); o quarto nível, pelas supervisões de departamentos; o quinto nível, pelas coordenações de áreas e o sexto nível, pelas lideranças de nível superior (enfermeiros, farmacêuticos, nutricionistas).

A partir dos depoimentos nas entrevistas, podemos perceber que, para diferentes tipos de cargos dentro da organização, há diferentes características que distinguem os trabalhos. Para o trabalho rotineiro e repetitivo, identificados na função dos técnicos em enfermagem, por exemplo (T1), encontramos alto nível de formalização (+F), com normas e procedimentos escritos ou definidos no sistema de informações implantado na organização; alto nível de centralização com uma única chefia, cuja tomada de decisão está diretamente relacionada a essa chefia, caracterizando alta centralização (+C) de suas tarefas; baixo nível de especialização (-K), sendo que o segundo grau completo e conhecimento em informática básico servem para o nível de técnicos; as políticas de gestão de pessoas (GP) são as mesmas para todos os cargos. Assim, conforme depoimento da gestora de $\mathrm{RH}$, as funções de recrutamento, seleção e capacitação são estruturadas na organização, sendo que as mesmas estarão presentes nos diferentes tipos de trabalho, desde T1 (rotineiro e repetitivo) até T4 (qualificado e inovador), embora, para T3 (profissional) e T4, tenhamos encontrado algumas peculiaridades, como os incentivos, que são oferecidos aos profissionais conforme sua avaliação. Assim, entendemos que as políticas funcionais existem e as complementares ainda estão em desenvolvimento, pois encontramos altos níveis de políticas funcionais (+GP) e não se encontraram evidências de políticas complementares.

Para o trabalho não rotineiro e qualificação média (T2), considerando os diferentes níveis de intensidade (maior ou menor) de todos os componentes de desenho (+C, -F, -K, -GP) indicados na teoria, não se conseguiu caracterizá-lo na organização em função da necessidade de baixa formalização, característica desse tipo de trabalho. Tendo em vista que o hospital segue um padrão de formalização muito elevado para todas as atividades, devido às exigências dos diferentes protocolos que deve seguir, desde os referentes à higiene e limpeza, garantindo a segurança contra infecções, até os padrões da profissão (médicos e enfermeiros), esta composição (-F) não se apresenta em nenhum momento. Portanto, as condições para ocorrer T2 levam a encontrar sempre T1, com alta formalização, alta centralização, baixa especialização e baixas políticas de gestão de pessoas.

Para o trabalho profissional (T3), encontramos a caracterização do alto nível de formalização (+F), com a identificação de vários mecanismos, como reuniões de enfermeiros, comunicação interna formalizada em e-mail, cartilhas, mural e por meio de um dossiê profissional, em que constam quais são os tópicos que se deve seguir e atingir. Quanto à centralização, o nível é baixo (-C) nesta função (enfermeiro), pois seguem a supervisão nas decisões estratégicas de como desenvolver as atividades de um enfermeiro, auxiliando na capacitação dos mesmos e caracterizando um baixo nível de centralização (-C). Quanto à especialização, podemos observar que o entrevistado possui um elevado grau de especialização (+K) e afirma que, para determinados cargos, como médico, fisioterapeuta, nutricionista, exige-se um alto nível de conhecimento específico como profissionais de nível superior e com experiência na área de terapia intensiva ou com pós-graduação preferencialmente.

Para o trabalho qualificado e inovador (T4), podemos observar que, embora haja alta formalização, exigida por toda a organização, há um diferencial para os médicos, caracterizando um menor nível de formalização (-F), segundo entrevista com a alta direção:

O hospital tem um guia ético que serve para todos. Cada categoria tem seu código ético e profissional, cada área tem a determinação das suas atividades. Mas (...) temos o corpo clínico que é uma entidade dentro do hospital que é formada pelos médicos que não são contratados do hospital. Então essa ação é um pouco limitada com esses profissionais.

Por meio deste depoimento, também podemos observar que, por não se tratar de médicos contratados do hospital, esses não têm um local específico de trabalho, podendo atuar em suas clínicas, no hospital e em outros setores. Sendo assim, esta é uma atividade de difícil medição e controle, caracterizando um baixo nível de centralização (-C). Considerando o conhecimento exigido na atuação do corpo clínico do hospital, podemos perceber que há um elevado nível de especialização (+K) para os médicos.

Quanto aos componentes do processo de formação da estratégia na organização, os níveis de racionalidade no processo de tomada de decisão são elevados (+R), podendo estar relacionados com os níveis de formalização, que são elevados em todos 
os tipos de trabalho, ainda que haja a especificidade de baixa formalização nas atividades do corpo clínico (encontrado em T4). A visão "Ser reconhecido como um centro de excelência no cuidado à saúde" é transmitida a todos os colaboradores de forma intensa, assim como a missão e os valores da organização (+V), evidenciados nos documentos apresentados.

A participação no processo de tomada de decisão ocorre por meio de reuniões periódicas (semanais) nos níveis mais elevados (+P). Por outro lado, todos os colaboradores têm oportunidade de participar em reuniões semestrais com a alta direção, como informou a supervisora de RH.

A flexibilidade, de acordo com Hamel e Välikangas (2003), está relacionada à capacidade de cada um se adequar às mudanças que ocorrem no ambiente interno para atender às exigências e necessidades do ambiente externo (clientes, fornecedores e usuários em geral). No hospital, a flexibilidade é alta ( $+\mathrm{Fx}$ ) quando se refere a trabalhos de baixa formalização (T4); no entanto, quanto às demais atividades que estão altamente formalizadas, o nível de flexibilidade é baixo (-Fx), tendo em vista a necessidade de seguir os protocolos rígidos estabelecidos.

A segunda organização pesquisada, Caso B, foi uma indústria têxtil, com mais de cem anos no mercado e que continua expandindo suas atividades tanto no âmbito nacional quanto internacional. A empresa está organizada hierarquicamente, sendo representada pelo conselho administrativo, estando logo abaixo a presidência. A diretoria é formada pelas diretorias: comercial; financeira; administrativa; industrial; cadeia de suprimento e logística; e marketing e produto, que se reportam à presidência e estão acima do nível gerencial composto por trinta gerências. As gerências coordenam o nível de supervisão e coordenações de setores compostos pelos níveis técnicos e operacionais. As entrevistas foram feitas nos níveis de direção, gerência, supervisão e operacional; podemos observar que os entrevistados tinham seus determinados locais de trabalho. Os trabalhadores do nível operacional trabalhavam no seu setor localizado no prédio de produção. Já os trabalhos considerados com maior nível hierárquico tinham suas respectivas salas no prédio administrativo, porém circulavam e trabalhavam por todo o edifício.

Para o trabalho rotineiro e repetitivo (T1), podemos identificar nas atividades padronizadas; conforme o depoimento de uma auxiliar de produção, quando disse: "Os tamanhos, a quantidade, está tudo no sistema. Está tudo formalizado. Tem que seguir aquela quantidade de serviço, passo a passo. Devido à rotina do setor de produção estar protocolada, tem que seguir rigorosamente o que é exposto", evidenciando que há um alto grau de formalização (+F).

Já a centralização foi observada nas visitas à organização e também por meio do relato da auxiliar de produção sobre o nível de tomada de decisão, que é seguida uma hierarquia e que cada um decide no seu nível; no entanto, existe o chamado DDSQ (diálogos diários de segurança e qualidade), no qual os trabalhadores podem apontar situações que necessitam de mudança e que são levadas para a gerência. O DDSQ ocorria diariamente, mas, com a evolução/melhoria do processo, passou a ocorrer semanalmente. Sendo assim, percebemos um nível médio de centralização (+/-C) nesse setor.

Podemos observar que é baixa (-K) a especialização que os funcionários precisam ter nesse setor, considerando que: "Tem funcionário que não tem o ensino médio, porque é mais serviço braçal. Mas o pessoal da costura tem curso, normalmente, vem de outras empresas, então tem experiência". Quanto à Gestão de pessoas (GP), verificamos que existe formalizado o recrutamento, a seleção e a capacitação:

Essa pessoa passa pelo RH e aí tem as instrutoras, que dão o suporte. Aí é feito um teste de uma hora. Se a pessoa se saiu bem, eu e a supervisão damos um visto. Se a pessoa passou no teste, a instrutora ainda fica mais uma semana com a pessoa.

Quanto aos incentivos: "Nós temos a participação no lucro semestral. Quando se atinge a meta em todo o setor de acabamento, (...) ganha ' $x$ ' por cento, se não atingiu, diminui uns dez por cento." Há também auxílios para estudo, onde a empresa paga uma porcentagem dos estudos dos funcionários, e há também uma verba para viagens relacionadas à aprendizagem do trabalhador. Com isso, podemos identificar um alto nível de gestão de pessoas (+GP). Ainda quanto à GP, podemos evidenciar que é alta para todos os tipos de trabalho desse T1 (rotineiro e repetitivo) até T4 (qualificado e inovador), talvez, como decorrência da idade da organização no mercado em que atua.

Para o trabalho não rotineiro e qualificação média (T2), foi possível identificá-lo nas atividades do auxiliar técnico, quando ocorrem situações de quebras de máquina ou de ausência de algum colaborador em que é necessário sair da rotina para 
resolver a situação, ou seja, como diz o auxiliar técnico: "quando a máquina quebra, tem que procurar um jeito de recuperar esta 'malha' pra atingir minha meta"; evidenciando uma situação de baixa formalização do trabalho (-F), tendo em vista que é preciso se adaptar à situação.

A centralização no T2 foi observada nas visitas à organização e também por meio do relato da auxiliar de produção, quando se refere às decisões que vêm da diretoria e devem ser cumpridas, evidenciando que a centralização é elevada, mas com a criação do DDSQ, já citada, essa centralização cede espaço à maior participação: "tem o DDSQ, que é uma discussão diária de qualidade e de segurança, onde é falado o que acontece no setor, os problemas, o que pode se atingir de meta". Sendo assim, tanto o que foi observado nas visitas quanto os depoimentos indicam um nível médio de centralização (+/-C).

Quanto à especialização (-K), pode se verificar que, assim como no T1, esta não é exigida na área de produção, sendo que há atividades em que os empregados estão buscando se qualificar, mas o cargo não exige: "inclusive existe dificuldade para encontrar alguém para trabalhar nos teares, falta mão de obra, meu setor deveria ter 45 pessoas e hoje trabalho com 30 ", informa a auxiliar técnica de produção.

Para o trabalho profissional (T3), podemos observar que o setor de controladoria possui um alto grau de formalização (+F), tendo em vista o nível de controle, normas e procedimentos a serem seguidos internamente e para fins fiscais. É um setor que tem uma baixa centralização $(-C)$, devido à necessidade de se elaborarem os orçamentos de todos os outros setores, à facilidade de acesso à informação nos diferentes níveis hierárquicos e à participação na tomada de decisões. Por fim, exige um grau elevado de especialização (+K) a partir do momento em que é preciso elaborarem vários dados técnicos e específicos de determinadas profissões (contador, administrador e economista).

Para o trabalho qualificado e inovador (T4), conforme o depoimento do gerente de vendas sobre o estabelecimento e alcance de metas, verificamos a presença de baixa centralização e formalização no desenvolvimento das suas atividades, pois, ao se tratar de um colaborador que tem o papel de fiscalização e tomada de decisão, e com um cargo que exige a necessidade de negociação/barganha, o profissional não pode estar atrelado a regras específicas da organização, que possam vir a limitá-lo a certas ações; assim tanto a centralização (-C) quanto a formalização (-F) são baixas. Quanto à especialização (+K), percebemos um médio/alto grau de especialização exigidos para atender à necessidade de se elaborarem indicadores e propostas estratégicas.

Quanto aos componentes do processo de formação da estratégia na organização, a racionalidade no processo de tomada de decisão é elevada (+R); no entanto, é percebido um aumento na participação em diversos setores (de T1 a T4). A visão "Ser reconhecida como a mais rentável e melhor gestora de marcas de vestuário" é transmitida a todos os colaboradores de forma intensa, assim como a missão e os valores de respeito aos clientes, sustentabilidade, integridade e ética, inovação e abertura a mudanças e comprometimento da organização (+V).

A participação no processo de tomada de decisão ocorre por meio de reuniões periódicas (semanais) chamadas de DDSQ, o que tem aumentado a participação, principalmente, nos setores de produção (+P). A flexibilidade, neste caso, está relacionada aos níveis de formalização encontrados. Assim como no Caso A, encontramos alta flexibilidade (+Fx) nos trabalhos que apresentaram baixa formalização (T2 e T4) e baixa flexibilidade (-Fx) nas atividades que apresentaram alta formalização.

A terceira organização, Caso C, Instituto Federal de Educação, Ciência e Tecnologia (IF), possui atualmente dez Campi distribuídos pelo Estado. Com a criação dos Institutos Federais, em 2008, passou ao status de Universidade, unindo-se a outras escolas de mesma vocação no Estado para formar o Instituto Federal.

Nessa condição, com um apoio mais sistemático da reitoria e um plano de expansão traçado, veio também a responsabilidade de desenvolver suas ações de forma a atender às vagas do Ensino Profissionalizante de nível Médio e às Licenciaturas. O IF oferece educação em todos os níveis, desde a formação inicial e continuada até a pós-graduação. Preferencialmente, busca o atendimento das demandas regionais de localização dos campi, com isso promove a interferência positiva na transformação da realidade social e econômica, contribuindo para desenvolvimento dos arranjos produtivos locais e regionais. A consolidação dos cursos passa a ser a grande realização, do ponto de vista do ensino, da pesquisa e da extensão, com a responsabilidade de reestruturar a própria instituição para aos novos desafios, do ponto de vista da gestão estratégica.

As evidências colhidas para o trabalho rotineiro e repetitivo (T1) mostram que esse foi identificado nos trabalhos ou serviços exercidos por alguns técnicos administrativos e de serviços terceirizados de limpeza e manutenção, ainda que esta última 
requeira alguma preparação em trabalhos relacionados com a manutenção das instalações, como pinturas e obras. Esses trabalhos estão formalizados ( $+F$ ) mediante normas, procedimentos padronizados e regras, e apresentam uma centralização alta $(+C)$. A existência de políticas funcionais de gestão de pessoas existe como em toda empresa pública (com contratação por meio de concurso público), e as políticas complementares de gestão de pessoas não foram identificadas (-GP). Este tipo de trabalho é de fácil supervisão e controle (-K).

Para o trabalho não rotineiro (T2), foi possível identificá-lo nas atividades de segurança da Instituição, serviço prestado por empresas subcontratadas, que executam as tarefas de segurança e, frente a novas (inesperadas) situações, devem ter condições de flexibilidade (-C); e, nas atividades dos auxiliares de secretaria, que desempenham tarefas diversas (atendimento aos alunos), com baixos níveis de formalização do trabalho (-F) por um lado, mas com alto nível de formalização do sistema (regras, normas, legislação do serviço público) (+F); e baixo nível de políticas de gestão de pessoas ou complementares (-GP), conforme previsto na teoria.

Para o trabalho profissional (T3), que não está submetido a mudanças e é de difícil análise/controle, com alto grau de conhecimento explícito e/ou tácito, quando incorpora capacitação profissional e/ou experiência acumulada relevante, foi identificado nas atividades dos técnicos administrativos de educação (TAE) com qualificação suficiente que, em cargos de chefias ou em seu posto de trabalho, desempenham um trabalho profissional. Na maioria dos casos, no IF, este trabalho apresenta baixa dificuldade de medição/controle, caracterizando-o próximo às características de T1. Quanto aos docentes, o maior número de servidores é graduado, especialista, mestre ou doutor. Os docentes não pesquisadores (sem mestrado ou doutorado) correspondem a um T3, enquanto ao cumprimento das atividades de docência; mas os docentes doutores, que dedicam uma parte de seu tempo à pesquisa, correspondem a um T4, ainda que a dimensão docente de suas atividades corresponda também a um T3.

Para o trabalho qualificado e inovador (T4), que incorpora um alto grau de conhecimento explícito e tácito relevante, pode ser identificado claramente no IF, no corpo de gestores, pró-reitores e reitor. Neste caso, a centralização é baixa (-C), os controles são indiretos, baseados nos protocolos estabelecidos pela própria instituição ou por controles e avaliações de órgãos governamentais como o Ministério da Educação e Cultura (MEC) ou Coordenação de Aperfeiçoamento de Pessoal de Nível Superior (CAPES). Outra forma de controle ocorre por meio da produção científica do corpo docente (publicações), que objetiva subsidiar projetos de pesquisa àqueles pesquisadores atuantes em grupos de pesquisa (- C).

Assim, para T1 e T2, a supervisão existe, e o controle é exercido $(+C)$, sendo que, em determinados momentos, os servidores têm autonomia dentro do trabalho que realizam, porque conhecem o que têm que fazer, seguindo uma característica de organização democrática. Podemos considerar que ocorre o mesmo com o trabalho T3 próximo a T1. Quanto ao T3, no caso dos TAE's, a autoridade só é exercida quando é necessário dentro de uma unidade administrativa ou entre diferentes níveis hierárquicos, quando se trata de um T3 desempenhado pelos gestores, ou seja, com baixa centralização (-C).

A estratégia do IF se formaliza por meio do Plano de Desenvolvimento Institucional (PDI); no entanto, notamos que a estratégia se forma nos diferentes níveis institucionais, caracterizando-se como democrática e participativa, por seu caráter de instituição formadora do conhecimento. A racionalidade pode ser expressa por meio dos níveis de planejamento formal instituídos em cada área (ensino, pesquisa e extensão), que ocorre seguindo a calendários e definições de prazos do MEC. $O$ grau de racionalidade é alto $(\mathrm{R}+)$, nesses casos, quando se refere aos procedimentos e cumprimentos de editais e projetos predeterminados no orçamento anual.

A visão "Ser referência em educação, ciência e tecnologia na formação de profissionais cidadãos comprometidos com o desenvolvimento da sociedade" é pouco difundida (-V), porém as ações do dia a dia seguem o que está estabelecido nessa Visão, a qual poderia ser transmitida com mais intensidade aos colaboradores, assim como a missão e os valores da organização, pois constam formalmente no PDI, mas sua disseminação é passiva (exposta).

A participação existe de forma importante (+P), ocorrendo em todos os âmbitos, todos os níveis podem participar do processo de tomada de decisão: dentro dos Conselhos de Campus, Conselho de Diretores e do Conselho Superior (Universitário), por meio dos representantes de estudantes, professores ou técnicos administrativos, introduzindo elementos emergentes no processo de formação da estratégia (MINTZBERG e WATERS, 1985). 
A flexibilidade está atrelada às condições impostas pelas normas federais de procedimentos, que seguem as características de impessoalidade, legalidade e transparência, fazendo com que as rotinas organizacionais sejam burocráticas e tornem o processo de tomada de decisão moroso e engessado, com excesso de procedimentos para cada decisão, diminuindo a possibilidade de adequação rápida às mudanças que ocorrem no ambiente interno para atender às exigências e necessidades do ambiente externo (clientes, fornecedores e usuários em geral). No IF, a flexibilidade é baixa (-Fx) para todos os tipos de trabalho, já que o nível de formalização é elevado, seguindo os protocolos rígidos estabelecidos, Quadro 2.

\section{Quadro 2}

\section{Relação dos Casos e Tipos de Trabalho Encontrados}

\begin{tabular}{|c|c|c|c|c|}
\hline Casos & $\mathrm{T} 1$ & $\mathrm{~T} 2$ & T3 & T4 \\
\hline A & Técnicos enfermagem & - & Enfermeiro/médico & Alta direção \\
\hline B & $\begin{array}{c}\text { Aux. Produção; } \\
\text { costureira }\end{array}$ & Aux. Técnico & Controladoria & Gerente vendas \\
\hline C & $\begin{array}{l}\text { TAE, serv. Limpeza/ } \\
\text { manutenção }\end{array}$ & $\begin{array}{c}\text { Segurança, } \\
\text { aux. secretaria }\end{array}$ & $\begin{array}{c}\text { TAE/docente não } \\
\text { pesquisador }\end{array}$ & $\begin{array}{c}\text { Docente pesquisador/ } \\
\text { reitoria }\end{array}$ \\
\hline
\end{tabular}

Fonte: Elaborado pelos autores.

\section{DISCUSSÃo DA QUESTÃo DE PESQUISA E PROPOSIÇÃo TEÓRICA}

Considerando a questão de pesquisa definida inicialmente, "como os componentes do desenho e do processo estratégico organizacionais se relacionam com os tipos de trabalho desempenhados pela organização?", e a proposição teórica (P) elaborada, "a organização apresenta os componentes do desenho organizacional e da estratégia de acordo com os tipos de trabalho que desempenha, sendo que a simplicidade ou complexidade do trabalho determinam o adequado nível de ajuste" (de acordo com $\mathrm{P}_{\mathrm{a}^{\prime}}, \mathrm{P}_{\mathrm{b}^{\prime}}, \mathrm{P}_{\mathrm{c}^{\prime}} \mathrm{P}_{\mathrm{d}}$ ); passamos a analisar comparativamente os casos.

Nos Casos A e B, encontramos média centralização da decisão relacionada com a supervisão do trabalho (ao invés de alta); e alto nível de políticas de gestão de pessoas, com atividades de recrutamento, seleção e capacitação bem definidas, ao invés de baixo, como sugerem Moreno, Peris e González (2001, p. 217); por outro lado, encontramos evidências de médio nível de participação no processo de tomada de decisão com alto nível de compartilhamento e disseminação da visão, ao invés de baixo, ou seja, características de um processo de formação da estratégia emergente ou integradora (ANDERSEN, 2004), sendo que o esperado seria a relação de trabalho rotineiro (T1) com estratégia deliberada (MINTZBERG e WATERS, 1985). Assim, entendemos que a proposição $\left(\mathrm{P}_{\mathrm{a}}\right)$ se cumpre parcialmente. No Caso $\mathrm{C}$, a proposição se cumpre totalmente, já que as evidências indicam T1 (rotineiro) encontrado com as características apontadas na teoria (PERROW, 1970).

Para $\left(\mathrm{P}_{\mathrm{b}}\right)$, com relação à análise do trabalho não rotineiro (T2), o Caso A não apresentou evidências de caracterização do mesmo, devido à alta formalização dos processos correspondentes a este tipo de organização (hospitalar). Assim como no Caso B, o T2 não foi caracterizado, pois encontramos médio nível de centralização (ao invés de alto) e alto nível de políticas de gestão de pessoas, ao invés de baixo, conforme previa Perrow (1970); e, quanto à estratégia, encontramos que a visão é disseminada em todos os níveis, ao invés de baixo nível de compartilhamento da visão, segundo a teoria de Moreno, Peris e González (2001), com médio/alto nível de participação (ao invés de baixo) e alto nível de flexibilidade (ao invés de baixo), podendo ser atribuído ao fato de ser uma organização centenária (indústria têxtil madura). No Caso C, o nível de formalização do sistema é alto (+F), mesmo tendo um nível de formalização da tarefa baixo (-F), no caso dos técnicos administrativos de secretaria de cursos; a centralização e políticas de gestão de pessoas correspondem à teoria; a racionalidade elevada 
encontrada é propícia para esse tipo de trabalho, ainda que a participação seja elevada na prática (+P), o que se ajusta parcialmente com a proposição.

Para os trabalhos do tipo profissional (T3), o Caso A e B confirmam a proposição teórica elaborada $\left(\mathrm{P}_{\mathrm{c}}\right)$ quanto aos componentes de desenho, com médios níveis de formalização; baixa centralização; altas políticas funcionais/complementares de gestão de pessoas e alta especialização; mas, quanto ao processo de formação da estratégia, encontramos alta Racionalidade (ao invés de baixa) e baixa Flexibilidade (ao invés de alta), caracterizando um processo deliberado de formação da estratégia (MINTZBERG e WATERS, 1985) quando, para T3 profissional, o esperado seria encontrar um processo mais participativo. No Caso C, a proposição se cumpre para os TAE's, mas não se cumpre para o cargo de docente; também não foi encontrada a baixa racionalidade, nem a alta flexibilidade, conforme previa Perrow (1970).

Para o trabalho inovador (T4), caracterizado por baixos níveis de formalização; baixo nível de centralização; alto nível de políticas funcionais/complementares de gestão de pessoas e alto nível de especialização se ajustando com baixo nível de racionalidade e alto de participação, visão e flexibilidade $\left(P_{d}\right)$, corresponde a um processo emergente/integrador de formação da estratégia, o que foi evidenciado nos três casos. Assim, se confirma essa proposição quanto aos componentes de desenho, embora tenha sido encontrado alto nível de racionalidade (ao invés de baixa - estratégia deliberada) em certos procedimentos, principalmente, no caso do IF, no que se refere a cumprimento de prazos junto aos órgãos externos (MEC ou CAPES), Figura 4.

Figura 4

Análise Comparativa da proposição nos três casos

\begin{tabular}{|c|c|c|c|}
\hline \multirow{2}{*}{ Proposição } & \multicolumn{3}{|c}{ Casos } \\
\cline { 2 - 4 } & A & B & C \\
\hline$P_{a}$ & $\mathrm{CP}$ & $\mathrm{CP}$ & $\mathrm{C}$ \\
\hline $\mathrm{P}_{\mathrm{b}}$ & $\mathrm{NC}$ & $\mathrm{NC}$ & $\mathrm{CP}$ \\
\hline $\mathrm{P}_{\mathrm{c}}$ & $\mathrm{CP}$ & $\mathrm{CP}$ & $\mathrm{CP}$ \\
\hline $\mathrm{P}_{\mathrm{d}}$ & $\mathrm{C}$ & $\mathrm{C}$ & $\mathbf{C}$ \\
\hline \hline
\end{tabular}

Fonte: Elaborado pelos autores.

Nota: Cumpre (C); Cumpre parcialmente (CP); Não se Cumpre (NC)

Assim, entendemos que a proposição se cumpre parcialmente no todo, pois se percebe que o processo de formação da estratégia é tanto de cima para baixo (mais racional) quanto de baixo para cima (mais participação), dando espaço à flexibilidade e tendo a visão compartilhada, no entendimento de estratégia integradora de Andersen (2004), mas não como prevê a teoria.

Mesmo que as características de cada tipo de trabalho estejam presentes em cada um dos casos (com exceção de T2 no caso A), a forma de administrá-los não foi encontrada conforme o previsto na proposição teórica. A justificativa pode estar na falta de conhecimento dos gestores, principalmente no Caso A e C, que são organizações de setores específicos (saúde e educação), nos quais o profissional de gestão tem sua formação em área técnica.

Por outro lado, percebemos que cada caso apresenta características específicas que se aproximam da proposição teórica, faltando um pequeno ajuste nos componentes de desenho e de formação da estratégia, que não ocorrem, justamente, pela falta de visão da organização como um processo. Nos três casos, percebemos que as teorias organizacionais revisadas não são desempenhadas na prática tal qual prevê a teoria. O adequado nível de ajuste (CHILD, 1997) é resultado do conhecimento e desenvolvimento de todas as variáveis aqui estudadas (LAVARDA, CANET e PERIS, 2011). 


\section{CONSIDERAÇÕES FINAIS}

O objetivo desta pesquisa foi identificar os distintos tipos de trabalho e a seguir verificar, dentro da gestão do trabalho, como os componentes do desenho e do processo estratégico organizacionais se relacionam com os tipos de trabalho desempenhados pela organização.

Foi possível identificar os distintos tipos de trabalho (T1, T2, T3 e T4), bem como analisar os níveis de centralização, formalização e especialização dos mesmos, relacionando-os com os níveis de racionalidade, visão e participação no processo estratégico de cada Caso. Encontramos que o T2 (não rotineiro, de qualificação média/baixa) não foi caracterizado, devidamente, no Caso $\mathrm{A}$, e foram encontradas variações do modelo teórico que não confirmam totalmente a proposição nos Caso B e C. Entendemos que esta variação do modelo está vinculada a variáveis não estudadas nesta pesquisa, como o fator cognitivo do nível gerencial (SCHNEIDER e ANGELMAR, 1993; KAPLAN, 2011), a mudança no ambiente interno e externo (TOLBERT e ZUCKER, 1996; JOHNSON, MELIN e WHITTINGTON, 2007) e questões políticas não explicitadas no processo de gestão (CHILD, ELBANNA e RODRIGUES, 2010), que precisam ser estudadas com maior aprofundamento.

A análise dos resultados indicou que as organizações estudadas apresentam distintos tipos de trabalho; entretanto, entendemos que o não cumprimento da proposição teórica ocorreu, pois as evidências apontaram para um processo de formação da estratégia tanto de cima para baixo (mais racional) quanto de baixo para cima (mais participação), dando espaço a flexibilidade e tendo a visão compartilhada, conforme entendimento de estratégia integradora de Andersen (2004), e não como prevê a teoria (Figura 2).

Mesmo que as características de cada tipo de trabalho estejam presentes em cada um dos casos, a forma de administrá-los não foi encontrada, conforme o previsto na proposição teórica, não se encontrando o ajuste (fit) adequado previsto (CHILD, 1997).

Assim, a contribuição deste estudo está em uma melhor compreensão da relação entre as variáveis envolvidas na gestão de diferentes tipos de trabalho e na forma como foi identificada e analisada a gestão dos componentes de desenho e do processo de formação da estratégia, permitindo responder à questão de pesquisa formulada inicialmente, ou seja, que cada organização apresenta os diferentes componentes do desenho organizacional e da estratégia e trata de administrá-los de forma peculiar, ainda que esta forma não seja aquela prevista na teoria.

A comparação dos casos apontou para a necessidade de se continuar fazendo este tipo de investigação in loco, buscando o aprofundamento da resposta à questão de pesquisa e novas categorias de análise que expliquem o fenômeno estudado, pois a adequada gestão dessas categorias é o desafio que se apresenta aos gestores, já que é complexo o delineamento e entendimento do maior ou menor nível de centralização, formalização, flexibilidade dentro da mesma organização para as diferentes atividades que executa.

Entendemos que a principal limitação deste estudo está relacionada à quantidade de entrevistas realizadas, pois somente alguns cargos foram analisados, o que procuramos minimizar com a análise de documentos e com observação direta que propiciou a triangulação dos dados.

Como futuras linhas de pesquisa, destacamos a necessidade de se analisarem outros cargos para ter uma abrangência e profundidade maior com relação aos tipos de trabalho encontrados, assim como investigar outras variáveis (ou categorias de análise) que possam explicar o aumento da participação e flexibilidade no processo de formação da estratégia, como a cognição (SCHNEIDER e ANGELMAR, 1993), a mudança no ambiente (TOLBERT e ZUCKER, 1996) e o papel do estrategista nesse processo (WHITTINGTON, 2014), por exemplo.

Por fim, esperamos que os resultados encontrados possibilitem uma contribuição aos gestores das organizações pesquisadas no sentido de que os mesmos passem a considerar a caracterização dos diferentes tipos de trabalho como fonte de conhecimento da própria organização e como forma de melhoria na tomada de decisões estratégicas, identificando as áreas onde deve haver maior ou menor controle, permitindo também definir os tipos de incentivos adequados a cada tipo de trabalho, assim como identificar os resultados que vêm sendo alcançados com a maior participação e menor racionalidade, que tem sido implementada no processo de formação da estratégia (JARZABKOWSKI, BALOGUN e SEIDL, 2010; BALOGUN, JACOBS, JARZABKOWSKI et al., 2013).

Como contribuição acadêmica, destaca-se a importância deste estudo para a linha de pesquisa de estratégia e de gestão de pessoas tão necessária e apoiada por organismos fomentadores de pesquisa como o CNPq, como é este caso. Por outro lado, entendemos que os estudos em administração ainda requerem profundidade e análise interna (micro-organizacional) para as organizações se fortalecerem e enfrentarem as instabilidades, assim como a evolução no campo dos estudos de gestão do processo estratégico mais participativo que aparece nos casos analisados, conduzindo a novos estudos no campo da estratégia na prática. 


\section{REFERÊNCIAS}

ALCHIAN, A. A.; DEMSETZ, H. Production, Information Costs and Economic Organization. The American Economic Review, v. 62, n. 5, p. 777-795, 1972.

ANDERSEN, T. J. Integrating Decentralized Strategy Making and Strategic Planning Processes in Dynamic Environments. Journal of Management Studies, v. 41, n. 8, p. 1271-1299, 2004.

BALOGUN, J. et al. Placing strategy discourse in context: sociomateriality, sensemaking and power. Journal of Management Studies, v. 51, n. 2, p. 175-201, 2013.

BULGACOV, S. et al. Administração estratégica - teoria e prática. São Paulo: Atlas, 2007. 190 p.

CHILD, J. Strategic choice in the analysis of action, structure, organizations and environment: retrospect and prospect. Organizational Studies, v. 18, n. 1, p. 43-76, 1997.

CHILD, J.; ELBANNA, S.; RODRIGUES, S. The Political Aspects of Strategic Decision Making. In: NUTT, P. C.; WILSON, D. (Eds.). Handbook of Decision Making. Chichester: Wiley. Cap. 5, 2010. 105-148 p.

CURY, A. Organização e métodos: uma visão holística. 7. ed. São Paulo: Atlas, 2000.

DAFT, R. L. Organizações: Teorias e projetos. São Paulo: Pioneira Thomson Learning, 2002. $532 \mathrm{p}$.

EISENHARDT, K. M. Building theories from case study research. Academy of Management Review, v. 14, n. 4, p. 532-550, 1989.

DENZIN, N.; LINCOLN, Y. O Planejamento da pesquisa qualitativa. 2. ed. Porto Alegre: Artmed, 2006. 432 p.

ELBANNA, S. Strategic Decision-Making: Process Perspectives. International Journal of Management Review, v. 8, n. 1, p. 1-20, 2006.

FARJOUN, M. Towards an Organic Perspective on Strategy. Strategic Management Journal, n. 23, p. 561-594, 2002.

FENTON, A.; LANGLEY, C. Strategy as Practice and the Narrative Turn. Organization Studies, v. 32, n. 9, p. 1171-1198, 2011.

FREDRICKSON, J. W.; MITCHELL, T. Strategic Decision Processes: Comprehensiveness and Performance in an Industry with an Unstable Environment. Academy of Management Journal, v. 27, n. 2, p. 399-423, 1984.

GODOI, C. K.; BANDEIRA-DE-MELLO, R.; SILVA, A. B. Pesquisa qualitativa em estudos organizacionais: paradigmas, estratégias e métodos. São Paulo: Saraiva, 2006.

GOLSORKHI, D. et al. Cambridge Handbook of Strategy as Practice. Cambridge University Press, 2010. 1-20 p.

HAMEL, G.; PRAHALAD, C. K. Competing for the Future. Harvard Business Review, p. 122-128, jul./ago. 1994.

HAMEL, G.; VÄLIKANGAS, L. The Quest for Resilience. Harvard Business Review, p. 1-13, set. 2003.

HART, S. L. An Integrative Framework for Strategy-Making Processes. Academy of Management Review, v. 17, n. 2, 327-351, 1992.
JARZABKOWSKI, P. Strategic Practices: an Activity Theory Perspective on Continuity and Change. Journal of Management Studies, v. 40, n. 1, p. 23-55, 2003.

JARZABKOWSKI, P.; BALOGUN, J.; SEIDL, D. B. Strategizing - the challenges of a practice perspective, Human Relations, v. 60, n. 1, p. 5-27, 2007.

JARZABKOWSKI, P.; BALOGUN, J.; SEIDL, D. B. Activity theory approaches to studying strategy as practice. Cambridge handbook of strategy as practice. Cambridge (UK): Cambridge University Press, 2010. $127-140$ p.

JOHNSON, G.; MELIN, L.; WHITTINGTON, R. Micro Strategy and Strategizing: Towards an Activity-Based View, Journal of Management Studies, v. 40, n. 1, p. 3-22, 2003.

JOHNSON, G. et al. Strategy as practice: research directions and resources. London: Cambridge, 2007. 260 p.

KAPLAN, S. Research in Cognition and Strategy: Reflections on Two Decades of Progress and Look to the Future. Journal of Management Studies, v. 48, n. 3, p. 665-695, may 2011.

KERLINGER, F. N.: Metodologia da pesquisa em ciências sociais: um tratamento conceitual. São Paulo: E.P.U. EDUSP, 1979.

LAVARDA, R.; CANET-GINER, M. T.; PERIS-BONET, F. How Middle Managers contribute to Strategy Formation Process: Connection of Strategy Processes and Strategy Practices. Revista de Administração de Empresas, v. 50, n. 40, p. 358-370, out./dez. 2010a.

LAVARDA, R.; CANET-GINER, M. T.; PERIS-BONET, F. Integrative Strategy Formation Process and the Management of Complex Work a Microorganizational Analisys. BBR - Brazilian Business Review (English Edition. Online), v. 7, n. 3, p. 108-129, 2010 b.

LAVARDA, R.; CANET-GINER, M. T.; PERIS-BONET, F. Understanding how the strategy formation process interacts with the management of complex work. European Business Review, v. 23, n. 1, p. 71-86, 2011.

MINTZBERG, H. The Nature of Managerial Work. Harper and How: New York, 1973.

MINTZBERG, H.; WATERS, J. A. Of Strategies, Deliberate and Emergent. Strategic Management Journal, v. 6, n. 3, p. 257-272, 1985.

MORENO-LUZÓN, M. D.; PERIS-BONET, F. J.; GONZÁLEZ, T. Gestión de la Calidad y Diseño de Organizaciones: Teoría y Estudio de Casos. Madrid: Prentice Hall, 2001.

NONAKA, I.; TAKEUCHI, H. Criação de conhecimento na empresa: como as empresas japonesas geram a dinâmica da inovação. 8. ed. Rio de Janeiro: Campus, 1997.

PÉREZ-AGUIAR, W. El estudio de Casos. In: SARABIA, F. J. (Ed.). Metodología para la investigación en marketing y dirección de empresas. Madrid: Pirámide. 1999.

PERROW, C. A framework for the comparative analysis of organizations. American Sociological Review, v. 32, n. 2, p. 194-208, 1967. 
PERROW, C. Organizational analysis: a sociological view. London: Tavistock. 1970

SCHNEIDER, S. C.; ANGELMAR, R. Cognition in organizational analysis: Who's minding the store. Organization Studies, v. 14, n. 3, p. 347-374, 1993.

TOLBERT, P.; ZUCKER, L. The institutionalization of institutional theory. In: CLEGG, S. R.; HARDY, C.; NORD, W. R. (Eds.). Handbook of organization studies, $1996.175-190 \mathrm{p}$.
WHITTINGTON, R. Completing the practice turn in strategy research. Organization Studies, v. 27, n. 5, p. 613-634, 2006.

WHITTINGTON, R. Making strategy: the hard work of institutional innovation in an open professional field. In: EUROPEAN ACADEMY OF MANAGEMENT ANNUAL MEETING, Valencia, 14, 2014, Valencia. Proceedings ...Valencia, 2014.

YIN, R. K. Estudo de caso: planejamento e métodos. 2. ed. Porto Alegre: 2005.

Rosalia Aldraci Barbosa Lavarda 